\title{
Offset effect on the S-Bend structure losses and optimization of its size for integrated optics
}

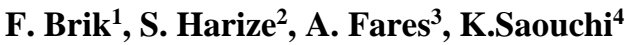 \\ 1,2,3,4 Department of Electronics, Engineering Sciences Faculty, University Badji Mokhtar-Annaba, Algeria \\ ${ }^{1,3,4}$ Laboratory of LERICA, Instrumentation and Communication of Annaba, University Badji Mokhtar-Annaba, Algeria \\ ${ }^{2}$ Laboratory of Automatic and Signal of Annaba (LASA), University Badji Mokhtar-Annaba, Algeria
}

\begin{tabular}{l} 
Article Info \\
\hline Article history: \\
Received May 28, 2019 \\
Revised Feb 28, 2020 \\
Accepted Mar 8, 2020 \\
\hline Keywords: \\
Integrated optics \\
Offset \\
Radius of curvature \\
Simulated technique \\
\hline
\end{tabular}

\begin{abstract}
The S-Bend structures are heavily exploited to join optical components. Reducing the power loss caused by the curve is the main objective in the design step of these components. However integrated optical circuits require S-Bend waveguide to be low loss and compact sized. In this paper, we present a contribution to link the curved structure to the straight waveguide by using the simulated bend function available in the Beam propagation tool of the Rsoft commercial software package. Simulation results confirm that this approach allows a reduction of the size of the curved structure with offset with relatively minimum of losses for photonic field.
\end{abstract}

Copyright (C) 2020 Institute of Advanced Engineering and Science. All rights reserved.

\section{Corresponding Author:}

Fatima Brik,

Department of Electronics,

LERICA Laboratory of Annaba,

University Badji Mokhtar-Annaba, Annaba, 23000, Algeria.

Email: fbrik2002@yahoo.fr

\section{INTRODUCTION}

The rapid development of optical fiber technology in telecommunications has required a growing need for active and passive integrated optical components. Waveguides represent the base of most of the integrated circuits to improve their performance while reducing the components dimensions, but the curvature of the waveguides leads to a power loss and a constraint on a transmission ability. The S-Bend structures are widely used to combine different elements of a circuit in order to build a variety of optical component like splitter [1], lasers [2,3], couplers [4, 5], switches [6, 7] and modulators [8-10]. Many solutions have been suggested to decrease the losses, but to lessen bending loss, the bending radius has to be increased. This will make the total size of the photonic integrated circuits larger, thing that is not suitable to the integrated optic.

The first proposition of lateral waveguide shift for loss reduction has been presented by Marcuse and Kitof [11, 12], though only the transition bending loss was considered. Another alternative study on a rib waveguide is presented in [13-15]. In [16-19] it was theoretically proposed an optimization method using a spline bend, which reduces the bending loss but requires a large footprint. Recently, the application of the bend composed of cloithoid curve has been demonstrated [20,21]. It is noted that in the above works, different approaches, methods and materials are used depending on the desired application.

In this paper, the effect of the different offset in the curved structure is investigated and a performance evaluation of the S-Bend is carried on with the Beamprob simulator integrated in the Rsoft software. The paper points up radiation and transition losses as the main sources of the propagation losses. The first are related to light going out of the waveguides during the propagation at the S-bend path, while the second is associated to the loss produced when the S-Bend are attached to the straight output waveguide.

Using Simulated Technique Bend, which is the appropriate method for the modeling of a single structure or a series of curves, we established the presence of the existence of two types of losses: pure bending 
losses (radiation) and transition losses [22]. Thereafter, an approach leading to transition losses reduction is proposed. It consists on the introduction of a shift which allows centering the second guide on the maximum of the mode guide of the first. The paper is structured into two sections: In section 2, the simulated bend technique and

\section{ANALYSIS METHOD}

In this paper, the simulated Bend and the offset method are proposed to study the S-Bend Structure.

\subsection{Bend technique}

The necessary computation time of a curved waveguide direct simulation is very high. This is due to the fact that the simulation necessitates a finer grid and size domain. But, a different and appropriate way for the simulation of a curved structure is available in the BeamProb simulator. The computational core of this program is based on a Beam Propagation Method (BPM), which is the most widely used technique for modeling fiber optic and integrated optics devices [23]. The principle of this method is based on the decomposition of the propagation of an electromagnetic wave in a homogeneous medium over a distance $\delta z$. This technique uses finite difference methods to solve the wave equation. In addition, the program necessarily uses boundary conditions [24].

The problem of the physical propagation of the wave requires two key elements:

- The distribution of the refractive index, $n(x, y, z)$.

- The electric and magnetic field at the entry, $u(x, y, z)$.

The algorithm requires additional input parameters such as:

- A finite domain of computation: $\left(x_{\min }, x_{\max }\right),\left(y_{\min }, y_{\max }\right)$

- The size of the transverse grid, $\Delta x$ et $\Delta y$.

- The size in the longitudinal direction, $\Delta \mathrm{z}$.

Using the simulated bend technique, the calculated mode in the curved area is easily obtained. The bending method consists of transforming the simulated geometry of a curved waveguide into a straight waveguide by modifying the refractive index obtained by using a multiplication by a coefficient $(1+\mathrm{x} / \mathrm{R})$. It has been proved that this approach is very precise for a waveguide width well below the curvature radius $(\mathrm{W}<<\mathrm{R})$.

\subsection{The offset technique}

Generally, modes curved wave guide are displaced outside of the turn, so a mode can be shifted laterally in a guide with respect to each other in order to improve the alignment of the curved waveguide modes. At the junction between each segment of the S-Bend structure, the integral overlap between the input mode and the output mode will be calculated and optimized. A monitor is implemented to calculate the overlap of the propagation in the field structure and its mode as well.

First, the modes of each segment of the curved structure will be calculated using bend technique. Then, an optimization of each shift at the junctions of the waveguides will be implemented so that it returns the value of the overlap between the modes. To find the optimized matching mode offset at each segment junction, the integral overlap between the input and output modes will be calculated and then maximized. By definition, the field at the beginning of the structure will be the launch field. Therefore, the monitor will return the required value at this point and the BPM simulation should be set so that it does not actually propagate at all, but only calculates the first data point. By setting the launch position along X, the launch field can be offset in relation to the structure, and the optimal value can be found by scanning over the launch offset.

\section{DESIGN AND SIMULATION RESULTS}

\subsection{The curved structure}

Figure 1 shows the S-Bend structure modeled under the RSOFT software. It consists of a straight input waveguide of length Lin (in Red color), two arc waveguides with opposite curvature (yellow and blue colors), and straight output waveguide of length Lout (in Red color) respectively. The waveguides are made of Silica and they considered stepping index. The total length of the structure is given as [22]: $s(z)=2 R \gamma+$ Lin + Lout

At first, the wavelength corresponding to the structure function is chosen to be $1.55 \mu \mathrm{m}$ and its dimensions are: Difference index $(\Delta \mathrm{n}=0.015)$, Radius $(\mathrm{R}=1500 \mu \mathrm{m})$, Length of input and output straight waveguide $(\mathrm{Lin}=\mathrm{Lout}=300 \mu \mathrm{m})$, angle of curvature $\left(\gamma=17^{\circ}\right)$ and width of the waveguide $(\mathrm{W}=5 \mu \mathrm{m})$. The Beam Prob monitor displays the transmitted power in the total length of the structure. Figure 2 presents the simulation results obtained by the monitor of the Beamprop simulator, which allows to post the intensity of the electric field (a) and the losses in power in the different segments waveguides forming the curved structure (b), and this for $($ Radius $=1500 \mu \mathrm{m})$. 


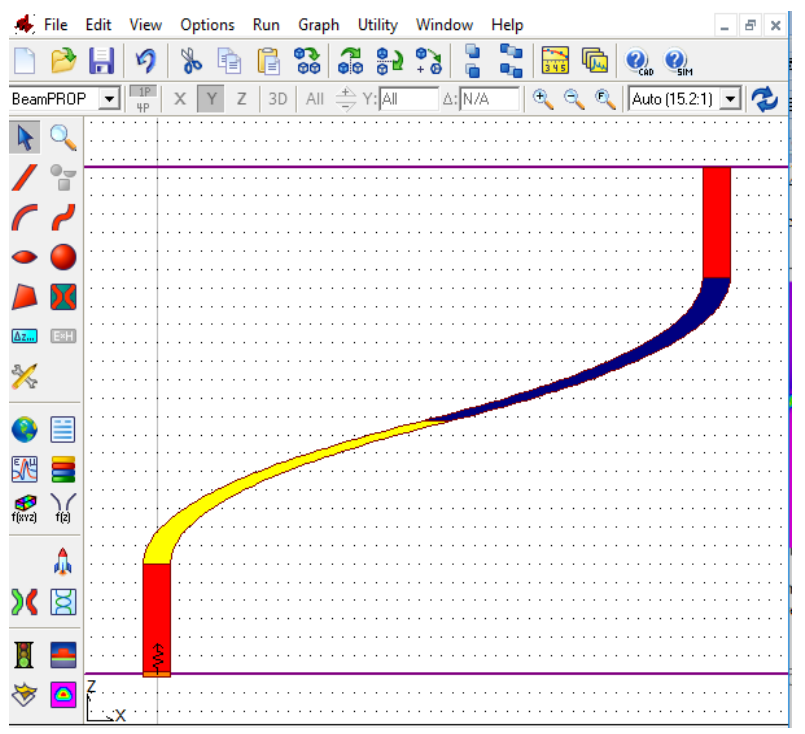

Figure 1. The S-Bend structure under software package
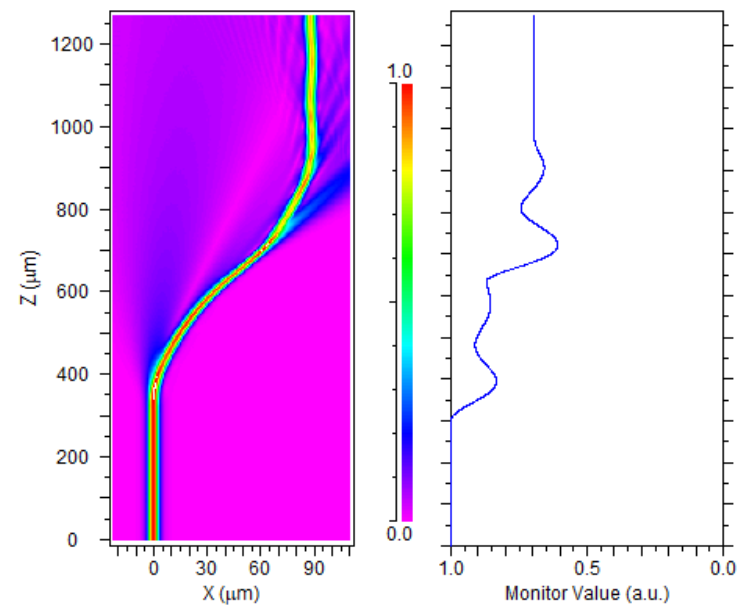

(a)

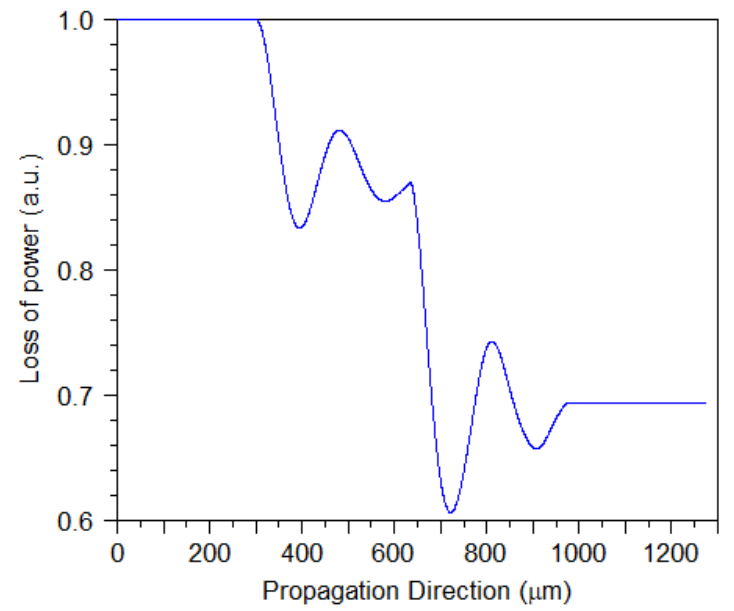

(b)

Figure 2. Losses of power in the total length of S-Bend without offset, (a) monitor intensity, (b) power loss in the total length of the structure

- The power transmission is estimated to $70 \%$ resulting in a loss of $30 \%$ for a radius of $1500 \mu \mathrm{m}$

From Figure 2, it can be observed that the transition losses between the two arcs of Radius curvature are more important than the other junction losses. To show the influence of these losses on the transmission of the power in the structure in S-Bend, we present the simulation obtained by considering a shift between the various segments of junctions.

\subsection{The curved structure with offset}

Actually, the presented structure has the same characteristics as the S-Bend one without offset, with the particularity of not aligned guides as demonstrated in Figure 3. The offsets noted (offset1, offset2 and offset3) represent the shift between the input guide-first curved guide, curved guide-curved guide- output guide and curved guide respectively, measured perpendicularly to the propagation direction $\mathrm{z}$ as shown in Figure 4 . The influence of the offset on the losses generated in a curvature structure has been examined to establish the best offset which enables the propagation of the optical power without deterioration. Since there are three segment junctions, the calculation will have to be performed three times. Using the offset method, we considered first only the offset between the two arcs (First curved guide-second curved guide) noted 
(offset $2=-0.9 \mu \mathrm{m})$ and (offset $1=$ offset $3=0$ ). In Figure 5, the transmission and losses of the shifted structure are shown. It can be seen that the transition losses between the two arcs are widely reduced by the value of the optimal offset.

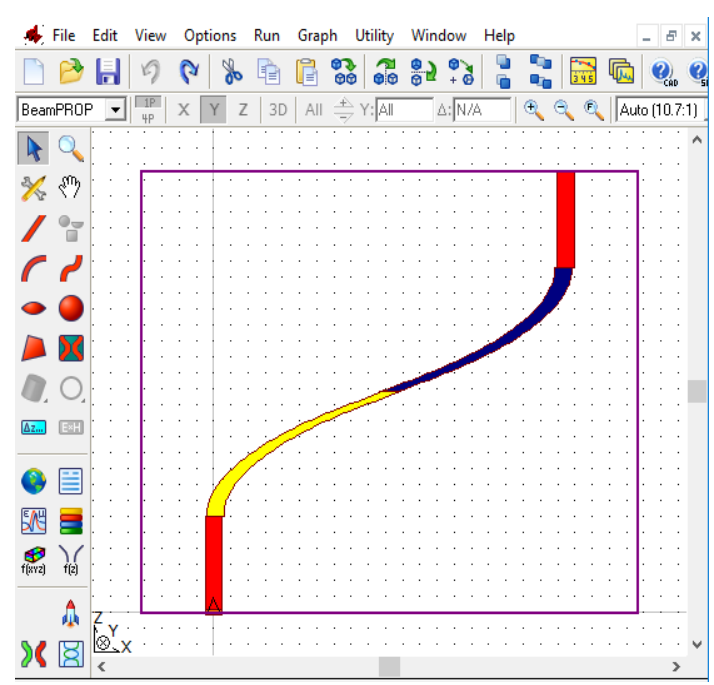

Figure 3. Sketch of the curved structure with shift under software package

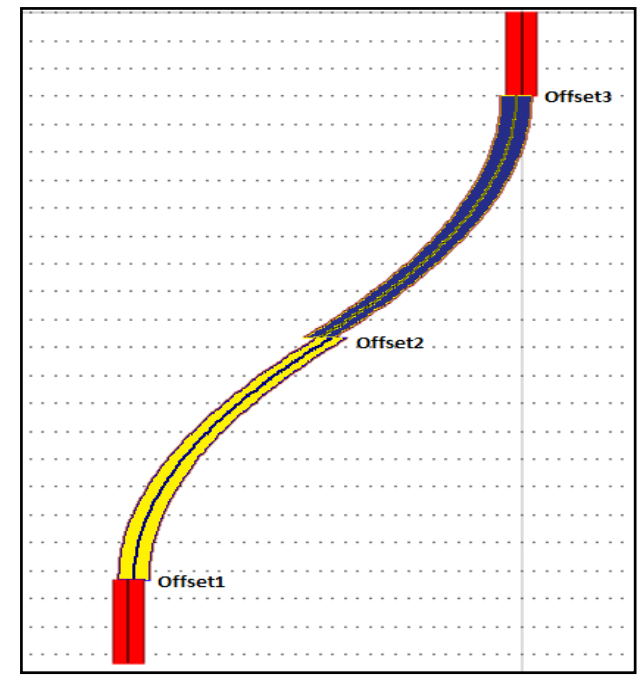

Figure 4. Position of the different offsets

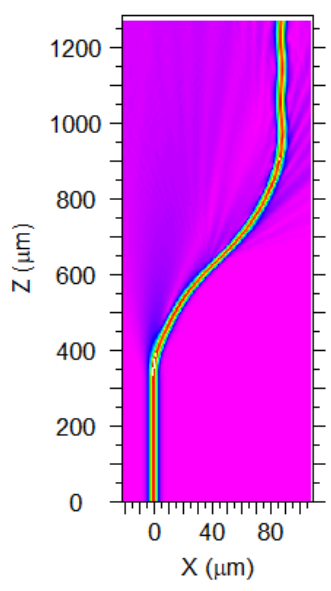

(a)
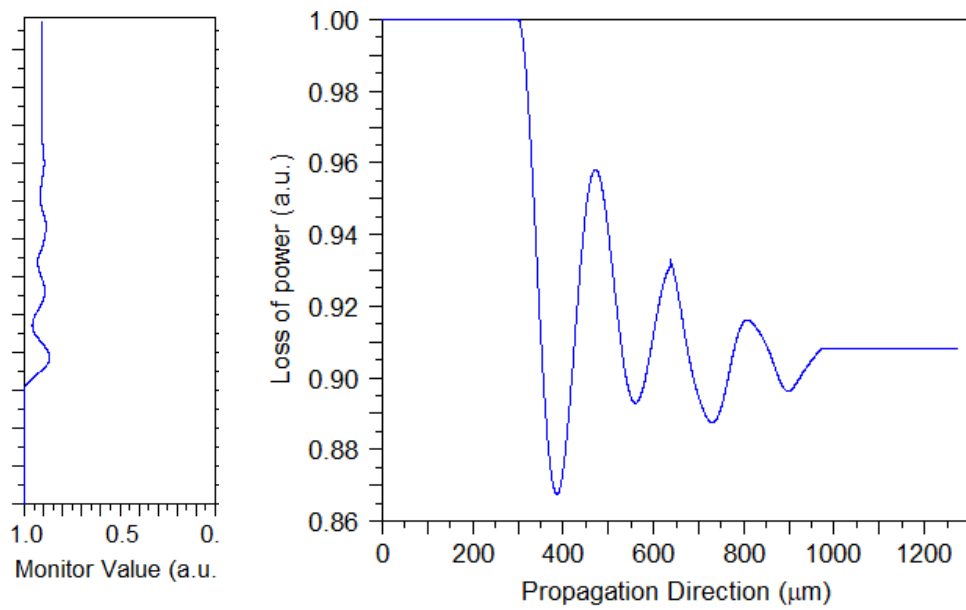

(b)

Figure 5. Normalized transmission of the shifted curved structure (offset $2=-0.9 \mu \mathrm{m}$ ), (a) monitor intensity, (b) power loss in the total length of the structure

- The power transmission is $91 \%$ resulting in a loss of $9 \%$ for a radii $\mathrm{R}=1500 \mu \mathrm{m}$.

In Figure 6, the normalized transmission (contour Map) and the power loss in the S-Bend structure for the offsets optimized offset 1 , offset 2 and offset 3 are displayed.

- The power transmission is $98.68 \%$ resulting in a significantly decreased loss of $1.32 \%$ for $\mathrm{R}=1500 \mu \mathrm{m}$.

Table 1 summarizes the different optimized parameters. For the shifted structure, the maximum power can be achieved for the optimal values of offset at the different junction, in contrast to the structure without offset. In consequence, the dimension of the curved structure is decreased to $1380 \mu \mathrm{m}$ which is compatible with integrated optics trends [25]. 

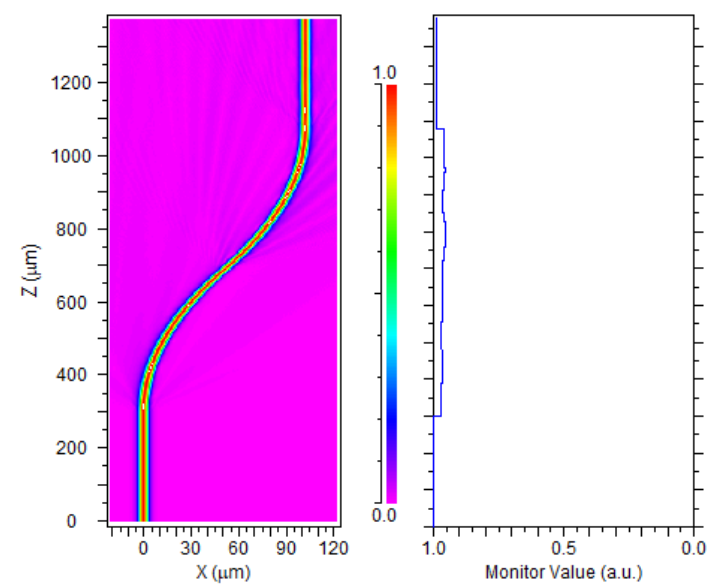

(a)

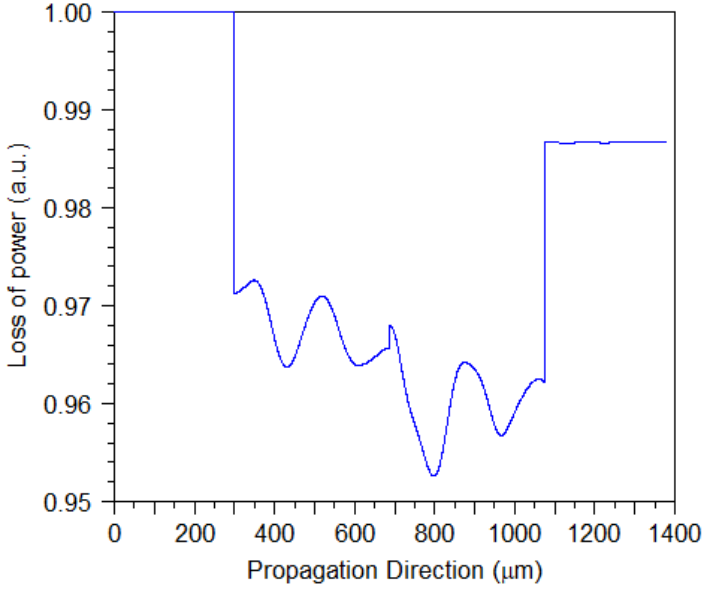

(b)

Figure 6. Normalized transmission of the shifted curved structure, (a) Monitor intensity,

(b) losses of power in the total length of the structure

Table 1. List of significant parameters of the shifted curved structure

\begin{tabular}{lc}
\hline Parameters & Values $(\mu \mathrm{m})$ \\
\hline Radius $(\mathrm{R})$ & 1500 \\
Length of the waveguide (Lin=Lout) & 300 \\
Offset 1 & 0.5 \\
Offset 2 & -0.9 \\
Offset 3 & 0.5 \\
Total length (S) & 1380 \\
\hline
\end{tabular}

\section{CONCLUSION}

In this paper, a technique available in the Rsoft software package called "Simulated Bend " has been shown and the results exposed. It has been put into evidence that there are two main loss sources. The first is due to the curvature radius, while the second occurs when the curved guide and the right guide modes have a spatial distribution and a dissimilar effective index when switching from one to another in the curved structure. Aiming at reducing the losses and improving the structure efficiency, the offset method has been introduced. By compensating the difference between the guide modes leading to an optimization of the curved structure dimensions. An evaluation of the curved structure with offset and without offset has been presented. It has been concluded that putting offset at the junction reduces the losses for the same level of the size. In perspective, it is intended to complete this work with experimental data and to investigate this structure based on photonic crystals to reduce its geometrical parameters as well as the various losses.

\section{ACKNOWLEDGEMENTS}

We are grateful to Mr. Mohamed Bouchemat, professor in Mentouri University, Algeria and Mr. Abdesslam. Hocini, professor in university of M'sila, for there fruitful discussion and help throughout this work.

\section{REFERENCES}

[1] Retno Wigajatri Purnamaningsih, Nyi Raden Poespawati, Elhadj Dogheche, "III-Nitride Semiconductors based Optical Power Splitter Device Design for underwater Application," International Journal of Electrical and Computer Engineering (IJECE), vol. 8, no. 5, pp. 3866-3874, October 2018.

[2] Mithaq M. Mehdy Al- Sultani, "Study the effect of thin film thickness on the optical features of (IR5 laser dye/CdSe nanoparticles) sample," TELKOMNIKA Telecommunication, Computing, Electronics and Control, vol. 17, no. 6, pp. 2877-2884, Dec. 2019.

[3] Mahmoud Tawfieq, Jorg Fricke, André Muller, Pietro Della Casa, Peter Ressel, Arnim Ginolas, Hans Wenzel, Bernd Sumpf and Gunther Trankle, "Characterisation and comparison between different S-bend shapped GaAs Y-Branch distributed Bragg reflector lasers emitting at 976nm, Semiconductor," in Semiconductor Science and Technology, vol. 33, no. 11, Sep. 2018. 
[4] Florian Dürr and Hagen Renner, "Analytical design for X couplers," Journal of Lightwave Technology, vol. 23, no. 2, pp. 876-885, Mar. 2005.

[5] Partha Pratim Sahu, P. P. Sahu, "Double S-bend structure for a compact two mode interference coupler," Applied Optics, vol. 50, no. 3, pp. 242-245, Jan. 2011.

[6] Nurdiani Zauhari, Abang Enmar Ehcan, Mohd Syuhaimi Abd Rahman, "S-Bend Silicon-On-Insulator (SOI) Large cross section Rib waveguide for directional coupler," International journal of Electrical and Computer Engineering (IJECE), vol. 7, NO. 6, PP. 3299-3305, Dec. 2017.

[7] F. Brik, N. Paraire, T. Bouchemat and M. Bouchemat, "Minimization of the thermal effect in a waveguide structure optical switch," Applied physics B, vol. 97, no. 4, 841-847, Dec. 2009.

[8] Fatima Brik, Abderraouf Fares, Fahima Fares, "Determination of different parameters of an optical switch structure with a metallic mirror," 6th International Conference on Computational and Experimental Science and Engineering (ICCESEN-2019) Antalya, TURKEY 23-27 Oct. 2019.

[9] Po Dong, Long Chen and Young-Kai Chen, "High-speed low voltage single drive push pull silicon Mach Zehnder modulators," Optic Express, vol. 20, no. 6, pp. 6163-6169, Mar. 2012.

[10] A. Musab, et al., "Enhancing the performance of strip and 180deg slot waveguide bends for integrated optical waveguide modulator," Optical engineering, vol. 58, no. 2, 2019.

[11] N. M. Ridzuan, M. F. L. Abdullah, M. B. Othman, M. B. Jaafar, "A Carrierless Amplitude Phase (CAP) Modulation format: Perspective and Prospect in Optical Transmission System," International Journal of Electrical and Computer Enginnering ( IJECE), vol. 8, no. 1, pp. 585-595, Feb. 2018.

[12] D. Marcuse, "Length optimization of an S-Shaped transition between offset optical waveguide transition," Applied optics, vol.17, no. 5, pp. 763-768, Mar. 1978.

[13] T. Kitoh, N. Takato, M. Yasu, M. Kawachi, "Bending loss in Silica based waveguide by using lateral offsets," in Journal of Lightwave Technology, vol. 13, no. 4, pp. 555-562, Apr. 1995.

[14] A. M. Shajakhan, Sheel Aditya, "Slope-matched S-bends for inclined integrated-optic waveguides," Microwave and optical Technology Letters, vol. 24, no. 4, pp. 267-271, Feb. 2000.

[15] Jared F. Bauters, Michael L. Davenport, Martijn J. R. Heck, J. K. Doylend, Arnold Chen, Alexander W. Fang, and John E. Bowers, "Silicon on ultra-low-loss waveguide photonic integration platform," Optics express, vol. 21, no. 1, pp. 544-555, Jan. 2013.

[16] Nurdiani Zauhari, Abang Enmar Ehcan, "Large cross section Rib Silicon- on-insulator (SOI) S-Bend waveguide," in Optik, vol. 130, pp. 1414-1420, Feb. 2017.

[17] Zhang Bing-na, Zhu Da-qing, Lei Wei, Zeng Si-hua, "Configuration optimization of S-shaped bend waveguide using B-spline," Chinese journal of laser, vol. 31, no. 11, 2004.

[18] Rupesh Kumar Navalakhe, Nandita Das Gupta, Buoy Krihna Das, "Fabrication and characterization of straight and compact S-Bend optical waveguides on a Silicon-on-insulator platform," Applied Optics, vol. 48, no. 31, pp. G125-G130, Nov. 2009.

[19] Zhen Hu, Ya Yan Lu, "Computing optimal waveguides bends with constant width," in Journal of Lightwave Technology, vol. 25, no. 10, pp. 3161-3167, Oct. 2007.

[20] G. Li, J. Yao, et al., "Ultralow-loss, high-density SOI optical waveguide routing for macrochip interconnects," Optics Express, vol. 20, no. 11, pp. 12035-12039, 2012.

[21] Fujisawa Takeshi, et al., "Low loss, compact, and fabrication-tolerant Si-wire $90^{\circ}$ waveguide bend using clothoid and normal curves for large scale photonic integrated circuits," Opt.Express, vol. 25, no. 8, pp. 9150-9159, Apr. 2017.

[22] Fatima Brik, Saliha Harize, Abderraouf Fares, "Reduction of an S-Bend structure size for optic telecommunication," 2019 IEEE International Conference on Design \& Test of Integrated Micro \& Nano-Systems (DTS), GammarthTunis, Tunisia, pp. 1-5, 2019.

[23] Y. Chung, N. Dogli, "An assessment of finite difference beam propagation method," in IEEE Journal of Quantum Electronics, vol. 26, no. 8, pp. 1335-1339, Aug.1990.

[24] Lallam Farah, Badaoui Hadjira, Abri Mehadji, "Design of a Selective Filter based on 2D Photonic Crystals Materials," International Journal of Electrical and Computer Engineering (IJECE), vol. 7, no. 4, pp. 1833-1838, Aug. 2017.

[25] Mehdi Zamani, Mansoureh Amanollahi, Abdesselam Hocini, "Photonic band gap spectra in Octonacci all superconducting aperiodic photonic crystals," Physica B: Condesed Matter, vol. 556, pp. 151-157, Mar. 2019. 